\title{
TIMP-1 mediates the inhibitory effect of interleukin-6 on the proliferation of a hepatocarcinoma cell line in a STAT3-dependent manner
}

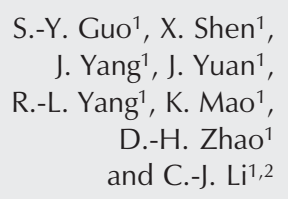

\section{Correspondence}

\section{C.J. Li}

Jiangsu Key Laboratory for

Molecular and Medical Biotechnology

Life Sciences College

Nanjing Normal University

Nanjing 210097

China

Fax: +86-25-8359-8812

E-mail: licj@njnu.edu.cn

Research supported by the Natural Science Fund of Jiangsu Province (No. 2000SWX000B501)

Received April 24, 2006 Accepted January 19, 2007 $\ldots \ldots \ldots \ldots \ldots \ldots \ldots . . \ldots$
1Jiangsu Key Laboratory for Molecular and Medical Biotechnology, Life Sciences College, Nanjing Normal University, Nanjing, China

${ }^{2}$ Animal Model Research Center, Nanjing University, Nanjing, China

\section{Abstract}

The tissue inhibitor of metalloproteinases (TIMP)-1 is a multifunctional protein which is not only an inhibitor of matrix metalloproteinases (MMPs) but also to have a possible "cytokine-like" action. Here, we first compared mRNA expression of TIMP-1 and MMP-9 in BEL7402 (a hepatocellular carcinoma cell line), L-02 (a normal liver cell line) and QSG-7701 (a cell line derived from peripheral tissue of liver carcinoma) using real-time quantitative RT-PCR. By evaluating the variation of the MMP-9/TIMP-1 ratio as an index of reciprocal changes of the expression of the two genes, we observed that the MMP-9/TIMP-1 ratio was about 13- and 5-fold higher in BEL-7402 than in L-02 and QSG-7701, respectively. Significantly, overexpression of TIMP-1 decreased the MMP-9/TIMP-1 ratio in BEL-7402 and then inhibited the cell growth to $60 \%$ and reduced the migration to about $30 \%$. Meanwhile, our data showed that interleukin-6 (IL-6) $(100 \mathrm{ng} / \mathrm{mL})$ could also inhibited the cell growth of BEL-7402. Further studies indicated that TIMP-1 mediated the inhibitory effect of IL-6 on BEL-7402 cell proliferation in a STAT3-dependent manner, which could further accelerate the expression of the cyclindependent kinase inhibitor p21. A dominant negative STAT3 mutant totally abolished IL-6-induced TIMP-1 expression and its biological functions. The present results demonstrate that TIMP-1 may be one of the mediators that regulate the inhibitory effect of IL-6 on BEL-7402 proliferation in which STAT3 signal transduction and p21 up-regulation also play important roles.

\section{Introduction}

Four tissue inhibitors of metalloproteinases (TIMP) have been identified; TIMP-1 has been shown to be particularly interesting. In addition to its classical role as a broad specific inhibitor of matrix metalloprotein-
Key words

- Tissue inhibitor of metalloproteinases-1

- Hepatocellular carcinoma

- Interleukin-6

- STAT3

- p21 ases (MMP) (1), TIMP-1 has also been reported to possess growth factor-like (2) and anti-apoptotic properties (3-5). TIMP-1 expression can be stimulated by a wide variety of agents including serum, growth factors, phorbol esters, cytokines, and viruses (6-8). It has been reported that the activation of 
hepatic stellate cells (HSCs), a key event in the pathophysiology of liver fibrosis (9), is also accompanied by induction of TIMP-1 activity and the expression of its mRNA (10, 11). It was shown that transgenic TIMP-1 inhibits simian virus $40 \mathrm{~T}$ antigen-induced hepatocarcinogenesis by impairing hepatocellular proliferation and tumor angiogenesis (12). All of these studies suggested that TIMP-1 might be involved in the process of liver pathogenesis.

The pleiotropic cytokine interleukin-6 (IL6) elicits a wide variety of biological activities in different cell types, including hematopoietic and neuronal cells, keratinocytes, osteoclasts, and vascular endothelial cells. IL-6 also modulates the hepatic expression of acutephase response genes during inflammation (13). When HSCs are activated, they secrete and respond to a wide range of cytokines and growth factors including IL-6. Furthermore, activated HSCs also secrete and deposit most of the excess extracellular matrix (ECM) in the fibrotic liver through increased secretion of ECM proteins, including TIMP-1 and TIMP2 (14). IL-6 has also been identified as a key protective factor from stress. During hyperoxic lung injury, IL-6 can protect lung cells from apoptosis by inducing the expression of Bcl-2 and TIMP-1 (15).

In the present study, we examined the functional involvement of IL-6 and TIMP-1 during liver carcinogenesis. Using a liver carcinoma cell line, we found that IL-6 was able to increase TIMP-1, that IL-6 and TIMP1 were able to inhibit cell proliferation, and that IL-6 induction of TIMP-1 and p21 expression was STAT3 dependent.

\section{Material and Methods}

\section{Antibodies and cytokine}

The recombinant human IL-6 was obtained from Merck Inc. (Whitehouse Station, NJ, USA). Rabbit polyclonal antibodies for TIMP-1 and B-tubulin were from Santa
Cruz Biotechnology, Inc. (Santa Cruz, CA, USA). All other chemicals were from Amersco Inc. (Kaysville, UT, USA).

\section{Plasmids and adenovirus}

Dr. David Young (Department of Cell Biology, Harvard Medical School, Boston, MA, USA) kindly provided the reporter plasmid pGL3-TIMP-1, in which the promoter region of human TIMP-1, spanning a fragment from -1714 to +17 bp was inserted before a luciferase reporter gene. The plasmids pcDNA3TIMP-1 and pEGFP-TIMP-1 were constructed in our laboratory. The plasmid p21(Waf1) luciferase and the control reporter plasmid pSV-ß3-galactosidase were gifts of Dr. ZhiMing Yin (Life Sciences College, Nanjing Normal University, Nanjing, China). The cDNA encoding STAT3 and dominant negative STAT3 were gifts from Dr. Xin-Yuan Fu (Department of Pathology, Yale University School of Medicine, New Haven, CT, USA) and were subcloned into expression vector pcDNA3.0 (pcDNA-STAT3wt and pcDNASTAT3cyf). STAT3cyf is a mutation of Stat3 tyrosine 705 to phenylalanine (Y705F) which causes a reduction of the tyrosine phosphorylation of wild-type STAT3 and inhibits the action of endogenous STAT3 in transfected cells (16). Dr. Xin-Yuan Fu also provided the M67 reporter plasmid in which STAT3 DNAbinding sites were inserted before a luciferase reporter gene.

Adenoviral vectors expressing human TIMP-1 were generated in our laboratory using the method described by $\mathrm{He}$ et al. (17). Briefly, the cDNA encoding full-length human TIMP-1 was cloned into the shuttle vector, pAdTrack-CMV, which contains a GFP expression cassette driven by a separate cytomegalovirus (CMV) promoter. pAdTrack CMV-TIMP-1 was recombined with backbone pAdEasy-1 in BJ5183 bacteria. Linearized recombinant plasmid DNA was then transfected into 293 cells, an adenoviral packaging cell line, to generate the recombinant adenovi- 
rus expressing TIMP-1 and GFP (Ad1-TIMP1). We used a GFP-expressing recombinant adenovirus (Ad1) as a parallel empty control.

The adenoviral backbone vector and shuttle vector were kindly provided by Dr. Tong-Chuan He (The Howard Hughes Medical Institute, Baltimore, MD, USA) (17). The simplified system described by He et al. (17) was used for adenovirus generation and amplification and titer determination. Viral particles were purified by cesium chloride density gradient centrifugation.

\section{Recombinant adenovirus infection}

Adenoviruses were incubated with cells in a small volume of serum-free medium at $37^{\circ} \mathrm{C}$. After adsorption for $2 \mathrm{~h}$, fresh complete growth medium was added and the cells were further incubated for the following experiments.

\section{Cell culture and transfection}

The BEL-7402, QSG-7701 and L-02 cell lines were purchased from the Shanghai Institute of Cell Biology, China, and were grown in Dulbecco's modified Eagle's medium (Gibco, Grand Island, NY, USA) supplemented with $10 \%$ NCS (HyClone, Hampton, NH, USA), 2 mM L-glutamine, $20 \mathrm{U} /$ $\mathrm{mL}$ penicillin, and $20 \mathrm{mg} / \mathrm{mL}$ streptomycin. The cultures were kept in a $5 \% \mathrm{CO}_{2}$ and $95 \%$ air humidified incubator at $37^{\circ} \mathrm{C}$.

Transfections were performed using the calcium phosphate method. All promoterluciferase fusions were co-transfected with the $\mathrm{pSV}$ - $\beta$-galactosidase plasmid to estimate transfection efficiency.

\section{Cell growth assay}

Subconfluent monolayer cells were trypsinized and plated onto a 6-well plate at a density of $10^{4}$ cells per well. After overnight post-plating, the cells were either treated with IL-6 (100 ng/mL), or infected with
Ad1-TIMP-1. The cell numbers were counted with a hemocytometer after trypsinization at different time points.

\section{Real-time quantitative RT-PCR}

Total RNA was isolated from cells using Trizol reagent (Invitrogen, Carsbad, CA, USA) according to the protocol of the manufacturer. Total RNA $(2 \mu \mathrm{g})$ was used for a reverse transcriptional reaction (Amersham Biosciences, Piscataway, NJ, USA). The primers were synthesized to amplify specific segments of the cDNA sequences of human TIMP-1, MMP-9, and B-actin; the $B-$ actin was used as an internal control. The primer sequences for each gene were as follows: TIMP-1 forward primer: 5' TTC CGA CCT CGT CAT CAG GG 3'; TIMP-1 reward primer: 5' ATT CAG GCT ATC TGG GAC CGC 3', MMP-9 forward primer: 5' CCT GGA GAC CTG AGA ACC AAT C 3'; MMP-9 reward primer: 5' GAT TTC GAC TCT CCA CGC ATC 3', actin forward primer: 5' TCC TGT GGC ATC CAC GAA ACT 3'; actin reward primer: 5' GAA GCA TTT GCG GTG GAC GAT 3'.

The qPCR assays were performed with the MyiQ SyBr Green Supermix system (BioRad, Hercules, CA, USA) and $300 \mathrm{nM}$ sense and antisense primers. The cycling conditions were as follows: initial denaturation at $95^{\circ} \mathrm{C}$ for $2 \mathrm{~min}$, followed by 40 cycles at $95^{\circ} \mathrm{C}$ for $30 \mathrm{~s}, 60^{\circ} \mathrm{C}$ for $30 \mathrm{~s}$, and $72^{\circ} \mathrm{C}$ for $30 \mathrm{~s}$. The expression level of each mRNA was determined relative to the standard curve using the LightCycler computer software (Roche Diagnostics, Castle Hill, NSW, Australia). The specificity of the SyBr Green assays was confirmed by melting point analysis and gel electrophoresis. The gene expression of B-actin was used for normalization.

\section{Western blotting analysis}

Cells were washed and lysed in lysis buffer (20 mM Tris- $\mathrm{HCl}, \mathrm{pH}$ 8.0, $75 \mathrm{mM} \mathrm{NaCl}, 1.5$ 


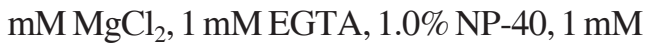
PMSF, $10 \mu \mathrm{g} / \mathrm{mL}$ leupeptin, and $10 \mathrm{mg} / \mathrm{mL}$ aprotinin). Protein concentrations were determined by Bradford assays. Samples of the extract containing equal amounts of total protein were submitted to $10 \%(\mathrm{w} / \mathrm{v})$ SDS-PAGE and transferred to the PVDF membrane, which was blocked with 3\% BSA-blocking buffer and then incubated with polyclonal anti-TIMP1 antibody diluted 1:500. The membrane was then incubated with the peroxidase-conjugated secondary antibody for $40 \mathrm{~min}$. The blotting patterns were developed using the ECL system (Roche, Mannhein, Germany). B-tubulin was blotted as a control of protein loading.

\section{ELISA}

The secreted TIMP-1 protein in culture medium was examined by indirect ELISA. Briefly, the wells of polystyrene microtiter plates (Greiner Bio-One, Maybachstrasse, Frickenhausen, Deutschland) were coated with $150-\mu \mathrm{L}$ culture medium after $72 \mathrm{~h}$ of infection with adenovirus. After overnight incubation at $4^{\circ} \mathrm{C}$, the wells were washed three times with 0.1 M PBS containing $0.05 \%$ Tween 20, pH 7.4. The coated wells were blocked with 200 $\mu \mathrm{L} 2 \% \mathrm{BSA}$ for $1 \mathrm{~h}$ at $37^{\circ} \mathrm{C}$ and then incubated with $150 \mu \mathrm{L}$ polyclonal antibody anti-TIMP-1 diluted 1:1000. After incubation for $2 \mathrm{~h}$ at $37^{\circ} \mathrm{C}$, the wells were washed as before and then incubated with $150 \mu \mathrm{L}$ of horseradish peroxidase-conjugated goat anti-rabbit IgG (1:5000 dilution, Sigma, St. Louis, MO, USA) for $1 \mathrm{~h}$ at $37^{\circ} \mathrm{C}$. After washing, $o$-phenylenediamine (Shanghai Chemicals, Shanghai, China) was used as the substrate. The reaction was stopped after 30 min with $50 \mu \mathrm{L} 2 \mathrm{M} \mathrm{H}_{2} \mathrm{SO}_{4}$ and absorbance was measured at $490 \mathrm{~nm}$ using an ELx800 Microplate Reader (Bio-Tek Instruments, Inc., Winooski, VE, USA).

\section{Luciferase assay}

BEL-7402 cells were transfected with the luciferase reporter construct $(0.2 \mu \mathrm{g} / \mathrm{each}$ well) when cells reached about $60 \%$ density on 6-well plates. At $24 \mathrm{~h}$ after transfection, cells were further stimulated with exogenous IL-6 $(100 \mathrm{ng} / \mathrm{mL})$ or infected with Ad1TIMP-1. Luciferase activities in cell lysates were determined with a luminometer (Lumat LB 9507, Berthold, Germany) 48 h after transfection, using a luciferase assay kit (Promega, WI, USA). Luciferase activity was normalized to ß-galactosidase activity to correct transfection efficiency. Each experiment was performed in triplicate and was repeated at least three times. $P$ values $\leq 5 \%$ were considered to be significant.

\section{Single-cell migration assay}

After pEGFP-TIMP-1 transfection, cells were plated onto a grid glass coverslip at low-cell density. In order to trace the movement of the cells, randomly selected fields were photographed every hour with a Leica digital camera. pEGFP-TIMP-1-expressing cells were distinguished under a fluorescence microscope and photographed with a Spot Cool CCD (Diagnostic Instruments, Sterling Heights, MI, USA). All pictures were merged together using the Photoshop software. The migration distance was compared between the "Green" cells (TIMP-1expressing cells) and normal cells.

\section{Motility assay}

The wounding assay was carried out according to the method described by Sato and Rifkin (18). Briefly, BEL-7402 cells were grown to confluence on 24-well plates in the presence of Ad1-TIMP-1 adenovirus, Ad1 control adenovirus, or mock control. Wounds were made in the monolayer by scraping it with a disposable pipette tip to remove a channel of cells. The cells were washed immediately with PBS and then cultured for 24 $\mathrm{h}$. The distance from the wound margin to the leading edge of the migrating BEL-7402 cells was determined. For each treatment, 
eight measurements were made at identical marked regions.

\section{Statistical analysis}

All experiments were repeated at least three times. Data are reported as means \pm standard deviation. Statistical differences between the two groups were evaluated using the paired Student $t$-test (Statistica, Statsoft Inc., Tulsa, OK, USA). P values less than 0.05 were considered to be significant.

\section{Results}

\section{Overexpression of TIMP-1-inhibited cell proliferation of BEL-7402}

BEL-7402 is an hepatocellular carcinoma (HCC) cell line extensively used in the
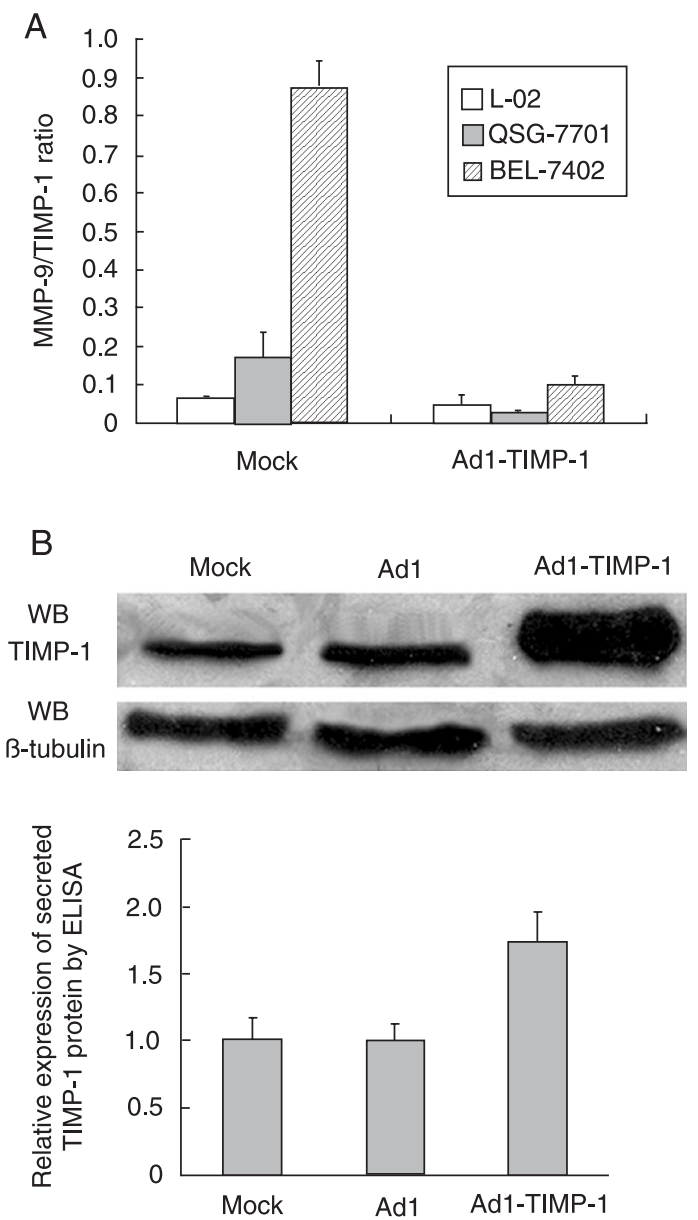

www.bjournal.com.br field of hepatoma research in China, while L-02 is a normal liver cell line used as a control and QSG-7701 is a cell line derived from peripheral tissue of liver carcinoma. Their genetic background, in particular regarding TIMP-1 expression, remains largely unknown. The result of real-time quantitative PCR showed that the expression of TIMP-1 tended to be decreased in BEL7402 cells compared to normal cell lines, whereas the amount of mRNA of MMP-9 presented an inverse trend. In addition, we evaluated the variation of the MMP-9/TIMP1 ratio as an index of reciprocal changes of the expression of the two genes and observed that the MMP-9/TIMP-1 ratio was significantly higher (about 13- and 5-fold, respectively, $\mathrm{P}<0.001)$ in BEL-7402 than in L-02 and QSG-7701, respectively (Figure 1A).

When cells overexpressed TIMP-1 by in-
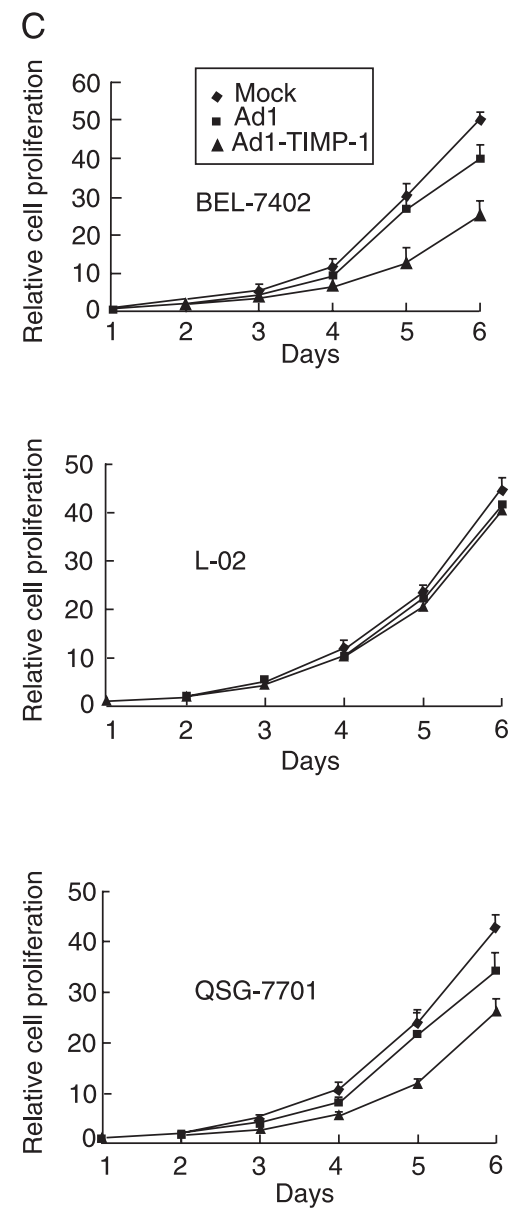

Figure 1. Overexpression of tissue inhibitor of metalloproteinases (TIMP-1) could inhibit the proliferation of the hepatocellular carcinoma BEL-7402 cell line. A, Comparison of the MMP9/TIMP-1 ratio in three different cell lines with recombinant adenovirus carrying TIMP-1 (Ad1TIMP-1) and without (mock) TIMP-1 overexpression. $B$, Detection of TIMP-1 overexpression in BEL-7402. Western blotting (WB) analysis was processed with ß-tubulin as internal control. The active protein of TIMP-1 secreted in the culture medium was detected by ELISA. The mock here indicated the cells without any treatment which was an internal control. C, Inhibition of BEL-7402, L-02 and QSG-7701 proliferation by TIMP-1. The cells were infected with Ad1-TIMP-1, Ad1 control and mock control. The number of cells was counted daily with a hemocytometer after trypsinization and the growth curves were calculated. 


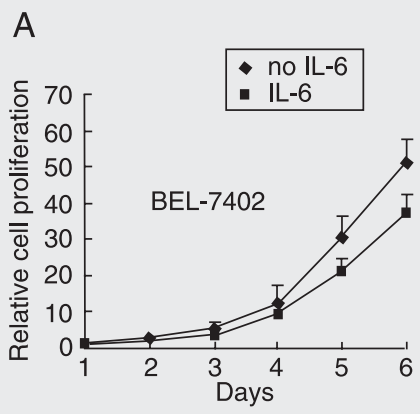

B

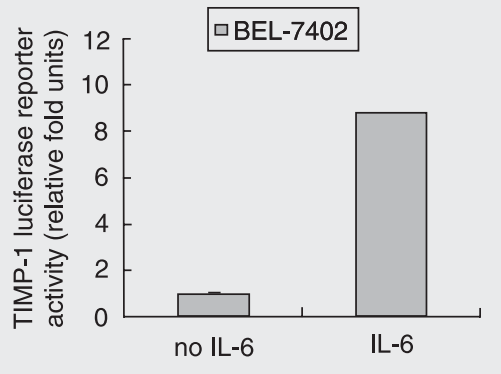

Figure 2. Inhibition by interleukin-6 (IL-6) of the proliferation of the hepatocarcinoma cell line BEL-7402 was accompanied by induced tissue inhibitor of metalloproteinases (TIMP-1) expression. A, BEL-7402 cells were collected by trypsinization and resuspended in culture medium after being treated or not with IL-6. The number of cells was counted with a hemocytometer. $B$, Cells were transfected with the TIMP-1 reporter plasmid and treated or not with IL-6. TIMP-1 expression induced by IL-6 was analyzed according to the luciferase activity of the TIMP-1 reporter construct. The B-gal reporter plasmid was co-transfected to normalize the transfection efficiency.

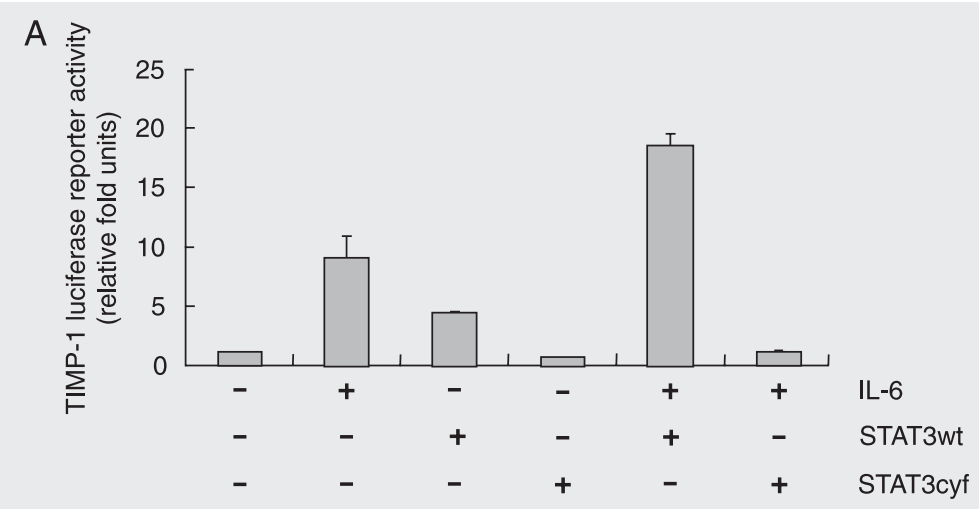

B

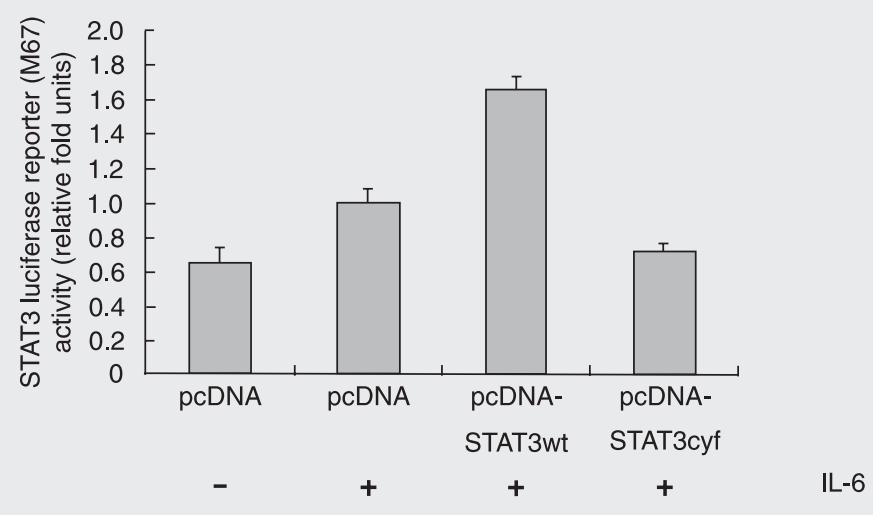

Figure 3. $A$, The induction of tissue inhibitor of metalloproteinases (TIMP-1) by interleukin-6 (IL-6) was STAT3 dependent, as demonstrated by a TIMP-1 luciferase reporter activity assay. Cells were transfected with TIMP-1 reporter plasmids, co-transfected together with the STAT3wt or STAT3cyf constructs. The cells were then treated or not with IL-6. B, Functional identification of STAT3wt/cyf protein using a STAT3 reporter (M67) activity assay. Cells were transfected with M67 reporter plasmids, co-transfected together with the pcDNA, pcDNASTAT3wt or pcDNA-STAT3cyf constructs. The cells were then treated or not with IL-6. Each group was co-transfected with the B-gal reporter plasmid to normalize the transfection efficiency. fection of recombinant adenovirus carrying the TIMP-1 gene, the MMP-9/TIMP-1 ratio was significantly decreased to almost the same level in BEL-7402, QSG-7701 and L-02 (Figure 1A). Cell growth was inhibited in BEL7402 and QSG-7701 cell lines (Figure 1C). The number of BEL-7402 cells declined to only about $60 \%$ of the number of untreated cells $(\mathrm{P}<0.01)$ after 6 -day culture by induction of TIMP-1, but L-02 was not observably affected by TIMP-1 overexpression. Interestingly, an obvious growth-inhibitory effect on QSG-7701 ( $\mathrm{P}<0.01)$ was also observed. The methyl thiazolyl tetrazolium (MTT) assay displayed similar results (data not shown).

\section{IL-6 inhibited the proliferation of the hepatocarcinoma cell line BEL-7402 and induced TIMP-1 expression}

Increasing evidence has demonstrated that IL-6 plays an important role in regulating cell proliferation. To investigate the role of IL-6 in the HCC cell line, we cultured BEL-7402 cells in a medium containing IL-6 $(100 \mathrm{ng} / \mathrm{mL})$ and found that cell proliferation was inhibited. After 6 days of culture, cell numbers decreased to only about $75 \%$ of untreated cells $(\mathrm{P}<0.05$; Figure 2A). This was also confirmed by the MTT assay (data not shown). The results also showed that IL- 6 only began to have visible effects after 4 days of treatment, which suggests that other factors induced by IL- 6 inhibited the proliferation of these cells.

To address whether the inhibitory effect of IL- 6 on BEL-7402 was accompanied by induction of TIMP-1, we checked the activity of luciferase that was under the control of the TIMP-1 promoter. The data suggested that IL-6 was able to induce TIMP-1 expression. The luciferase activity increased about 9-fold after IL-6 treatment (Figure 2B).

\section{IL-6 induction of TIMP-1 was STAT3 dependent}

Two functional binding sites for activa- 
tor protein-1 and STAT which responded to IL-6/oncostatin have been identified in the TIMP-1 promoter M (19). Here we examined whether STAT3 signaling was involved in TIMP-1 production induced by IL- 6 in BEL-7402. As shown in Figure 3, the TIMP1 reporter luciferase activity of BEL-7402 cells transfected with wild-type STAT3 expression vector was up-regulated about 4.4fold compared to control cells, and IL-6 treatment elevated TIMP-1 expression up to 18 -fold $(\mathrm{P}<0.01)$ in these cells. The dominant negative form of STAT3 totally inhibited the expression of TIMP-1 both in IL-6treated and -untreated cells. Thus, we found that the activation of TIMP-1 gene expression by IL-6 in BEL-7402 cells was dependent on STAT3 signaling.

\section{p21 was up-regulated by TIMP-1 and IL-6}

To elucidate the mechanism of IL-6-induced inhibition of cell proliferation in BEL7402, we checked the p21(Waf1) expression level using a luciferase reporter assay. IL-6 enhanced p21 expression about 3.3-fold compared to control cells, in which the transient overexpression of STAT3 augmented the luciferase reporter activity. When the cells were transfected with STAT3wt and treated with IL-6, they displayed up to 6.7 times more $\mathrm{p} 21$ expression than control cells. Moreover, dominant negative STAT3 blocked the stimulation of IL-6 to control levels (Figure $4 \mathrm{~A})$.

TIMP-1 itself was also able to accelerate p21 expression. The transfection of TIMP-1 resulted in a 5-fold higher expression of $\mathrm{p} 21$ than the vehicle vector transfection control and blank control (Figure 4B).

\section{TIMP-1 inhibited the migration of BEL-7402}

Both the single-cell migration detection assay and wounding assay showed that cells overexpressing TIMP-1 migrated much slower than control cells did. In Figure 5A, $a, b, c, d$ indicate the cell center at successive time points. The migration route of each cell can be considered to be $\mathrm{a} \rightarrow \mathrm{b} \rightarrow \mathrm{c} \rightarrow \mathrm{d}$ approximately, the average motion distance of the transfected cells (green cells) was only about $33 \%$ compared to the normal cells (Figure 5B).

The mobility of cell groups was detected by the wounding assay. Confluent monolayers of three groups of BEL-7402 cells were wounded and then cultured for $24 \mathrm{~h}$. The

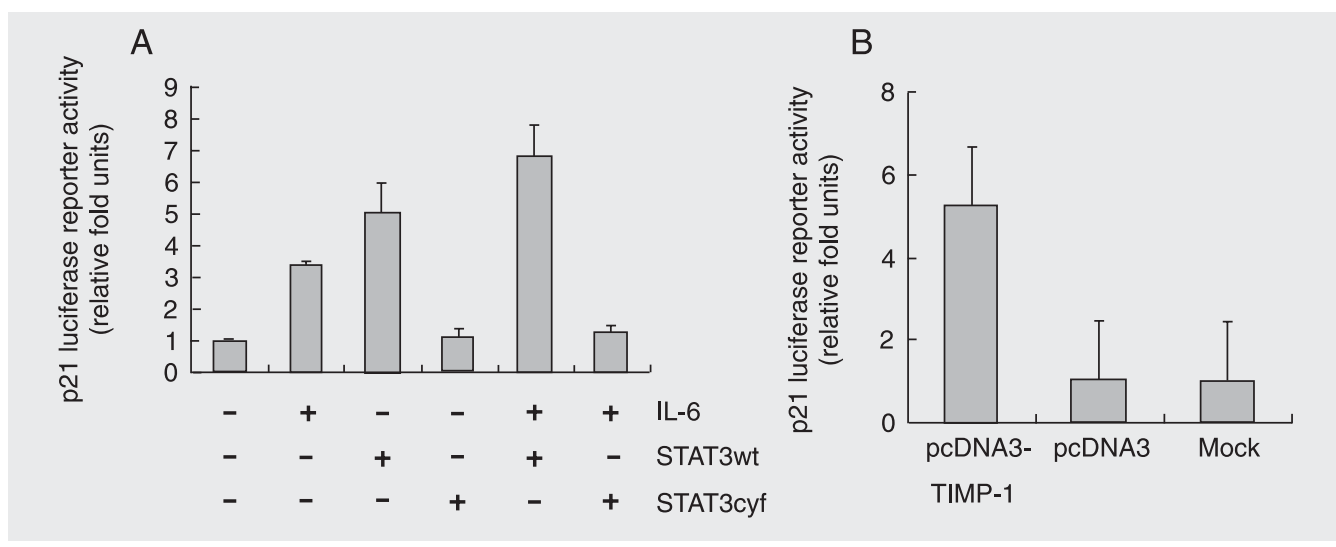

Figure 4. Up-regulation of p21 induced by tissue inhibitor of metalloproteinases (TIMP-1) and interleukin-6 (IL-6) in BEL-7402 cells analyzed by the luciferase assay. A, Cells were transfected with p21(Waf1) reporter, co-transfected together with pcDNA3-STAT3wt or pcDNA3-STAT3cyf constructs. The cells were then treated or not with IL6. B, Cells were transfected with p21(Waf1) reporter, co-transfected together with pcDNA3-TIMP-1 or pcDNA3 as an empty parental vector control. Mock was a blank control representing the cells transfected by nothing. Each group was co-transfected with the B-gal reporter plasmid to normalize the transfection efficiency. 
cells were photographed and the migration distance was determined (Figure 5C,D). The data showed that the migration rates of the Ad1-TIMP-1 group and the Ad1 group were 28 and $98 \%$, respectively, of the migration of the mock group. Both experiments indicated that TIMP-1 inhibited the migration of BEL-7402 cells.

\section{Discussion}

The ECM forms a microenvironment that can modulate cellular behavior by affecting the contact of the cell with the outside world $(20,21)$. Degradation or activation of ECM proteins by proteolysis can change the cellular microenvironment rapidly and irreversibly. The turnover of ECM is delicately regulated by a pair of contrary functional molecules: MMP and its tissue inhibitor (TIMP).
MMP produces subtle changes of matrix structure by cleaving and also releasing matrix-bound growth factors and thereby controlling differentiation.

MMP-9 has been reported to play an important role in the invasion and metastasis of cancers (22). A previous report showed that the MMP-9/TIMP-1 mRNA ratio differed between the stage II-III group and the stage IV group of lung cancer (23). Imbalance of the ratio between MMP-9 and TIMP1 may cause breakdown of the basement membrane and ECM and is an essential step in tumor invasion and metastasis (24). In the present study, BEL-7402 originated from a Chinese patient with $\mathrm{HCC}$ which exhibited a high metastasis rate. Real-time PCR data showed that the MMP-9/TIMP-1 ratio was noticeably higher in BEL-7402 than in the normal liver cell line L-02 and QSG-7701,
Figure 5. Inhibitory effect of tissue inhibitor of metalloproteinases (TIMP-1) on the migration of BEL-7402 cells. A, The centers of cells monitored every hour are shown by a, b, c, d. The cell on the right overexpressed TIMP-1. $B$, The relative average velocity of single cell migration was calculated for TIMP-1-transfected and control cells. $C$, Ability to migrate affected by TIMP-1 using a wounding assay. Confluent monolayers of three groups of BEL-7402 cells were scraped using a tip and then cultured for $24 \mathrm{~h}$. The cells were photographed and the migration distance was determined. The beeline indicated the same location. $D$, The migration distance of three groups was determined. ${ }^{*} \mathrm{P}<0.01$ for 7402/Ad1-TIMP-1 compared with the 7402/Ad1 group (Student $t$-test).

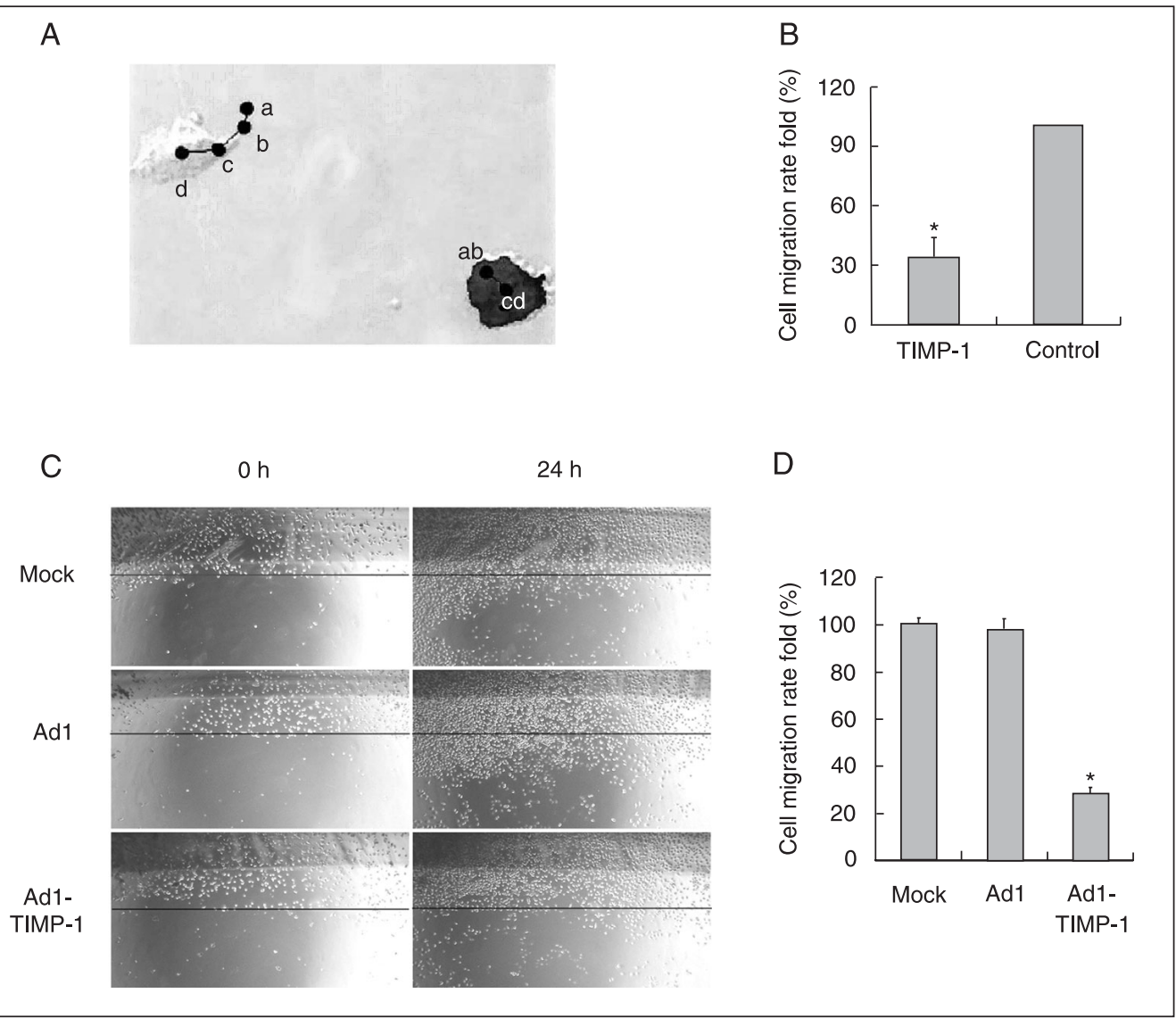


which may be one explanation for the high invasive ability of BEL-7402 compared to the other two lines.

TIMP-1 is a multifunctional protein with MMP-dependent and -independent actions for the regulation of cell death, cell proliferation, and angiogenesis (25). After TIMP1 was initially characterized as a homologue of erythroid potentiating activity factor, it was found to display growth- and survivalpromoting activity for a wide range of normal or transformed cells $(26,27)$. Increasing evidence demonstrates a much more complex role for TIMP-1 during tumor progression and angiogenesis, in addition to its regulation of MMP-mediated ECM degradation. It has been well established that TIMP-1 has anti-apoptotic activity on many different cell types through both MMP-dependent $(28,29)$ and -independent pathways (30,31).

Regarding the role of TIMP-1 in cell proliferation, conflicting data have been reported suggesting that TIMP-1 is mitogenic in certain cancer cells and lymphoid cells $(2,32)$ while it inhibits the growth of normal mammary epithelial cells (33). In the present study, TIMP-1 reduced the growth rate of the HCC cell line BEL-7402 by up-regulating the cyclin-dependent kinase inhibitor p21(Waf1) and reducing the MMP-9/TIMP1 ratio, suggesting that both MMP-9-TIMP1 balance and TIMP-1 expression as a "cytokine-like" protein are required for the regulation of tumor progression.

Previous studies have shown that IL-6 has a pivotal role in stimulating hepatocytes to produce acute phase proteins cytotoxic to the liver; moreover, IL-6 has been shown to be a cell cycle progression factor for liver cells (34). After 70\% partial hepatectomy, liver regeneration is dramatically impaired in IL-6 ${ }^{-/-}$livers. Treatment with IL-6 clearly accelerated the proliferative response in both IL- $6^{+/+}$and IL-6 $6^{-/-}$livers (35). Intense research over the past decade has shown that IL- 6 could inhibit the proliferation of some cancer cells (36-38). Zeng and Fan (39) suggested that IL- 6 could inhibit the growth of the hepatocarcinoma cell line BEL-7402 by increasing the influx of $\mathrm{Ca}^{2+}$ and decreasing the expression of Bcl-2 in the cell.

Acting via gp130, IL-6 activates multiple signaling pathways, including the JAKSTAT, the Src family of protein tyrosine kinases, and phosphatidylinositol 3-kinase. It is also known that IL-6 can induce TIMP1 expression (40). As indicated in a previous paper, IL-6 inhibits the proliferation of BEL7402 by promoting apoptosis (39). We found that IL-6 treatment did not inhibit BEL-7402 proliferation until the fourth day of treatment, suggesting that there might be another mediator induced by IL-6 which actually exerts the inhibitory effect. Our data showed that TIMP-1 may be the mediator of the inhibitory effects of IL-6, inducing the remodeling of the ECM. Actually cell growth and proliferation result from a balance between the number of dividing cells and the number of dying cells. And since TIMP-1 has been shown to inhibit apoptosis, our results suggested the presence of a potential downstream pathway regulated by TIMP-1 distinct from the anti-apoptosis pathway.

The present results showed that IL-6 induced cell growth inhibition through TIMP1 production, which was STAT3 dependent. The downstream inhibitory molecule of IL6 and TIMP- 1 was p21. IL- 6 could accelerate $\mathrm{p} 21$ expression through the STAT3 signaling pathway which may act by decreasing MMP activity.

\section{Acknowledgments}

We are grateful to Dr. David Young (Department of Cell Biology, Harvard Medical School, Boston, MA, USA) and Dr. TongChuan He (The Howard Hughes Medical Institute, Baltimore, MD, USA) for their generous gift of the construct of the pGL3TIMP-1 reporter plasmid or adenoviral backbone and shuttle vectors. We would like to thank Dr. Xin-Yuan Fu (Department of Pa- 
thology, Yale University School of Medicine, New Haven, CT, USA) for providing STAT3 wild-type, dominant negative form constructs and M67 reporter plasmid, and thank Dr. Zhi-Ming Yin (Life Sciences Col- lege, Nanjing Normal University, Nanjing, China) for providing the p21(Waf1) luciferase plasmid and the control reporter plasmid pSV-ß-galactosidase.

\section{References}

1. Denhardt DT, Feng B, Edwards DR, Cocuzzi ET, Malyankar UM. Tissue inhibitor of metalloproteinases (TIMP, aka EPA): structure, control of expression and biological functions. Pharmacol Ther 1993; 59: 329-341.

2. Hayakawa T, Yamashita K, Tanzawa K, Uchijima E, Iwata K. Growthpromoting activity of tissue inhibitor of metalloproteinases-1 (TIMP1) for a wide range of cells. A possible new growth factor in serum. FEBS Lett 1992; 298: 29-32.

3. Alexander CM, Howard EW, Bissell MJ, Werb Z. Rescue of mammary epithelial cell apoptosis and entactin degradation by a tissue inhibitor of metalloproteinases-1 transgene. J Cell Biol 1996; 135: 1669-1677.

4. Boudreau N, Sympson CJ, Werb Z, Bissell MJ. Suppression of ICE and apoptosis in mammary epithelial cells by extracellular matrix. Science 1995; 267: 891-893.

5. Guedez L, Courtemanch L, Stetler-Stevenson M. Tissue inhibitor of metalloproteinase (TIMP)-1 induces differentiation and an antiapoptotic phenotype in germinal center B cells. Blood 1998; 92: 13421349.

6. Campbell CE, Flenniken AM, Skup D, Williams BR. Identification of a serum- and phorbol ester-responsive element in the murine tissue inhibitor of metalloproteinase gene. J Biol Chem 1991; 266: 71997206.

7. Uchijima M, Sato H, Fujii M, Seiki M. Tax proteins of human T-cell leukemia virus type 1 and 2 induce expression of the gene encoding erythroid-potentiating activity (tissue inhibitor of metalloproteinases1, TIMP-1). J Biol Chem 1994; 269: 14946-14950.

8. Gewert DR, Coulombe B, Castelino M, Skup D, Williams BR. Characterization and expression of a murine gene homologous to human EPA/TIMP: a virus-induced gene in the mouse. EMBO $J$ 1987; 6: 651-657.

9. Friedman SL. Seminars in medicine of the Beth Israel Hospital, Boston. The cellular basis of hepatic fibrosis. Mechanisms and treatment strategies. N Engl J Med 1993; 328: 1828-1835.

10. Nieto N, Dominguez-Rosales JA, Fontana L, Salazar A, ArmendarizBorunda J, Greenwel P, et al. Rat hepatic stellate cells contribute to the acute-phase response with increased expression of alpha1(I) and alpha1(IV) collagens, tissue inhibitor of metalloproteinase-1, and matrix-metalloproteinase-2 messenger RNAs. Hepatology 2001; 33: $597-607$.

11. Bahr MJ, Vincent KJ, Arthur MJ, Fowler AV, Smart DE, Wright MC, et al. Control of the tissue inhibitor of metalloproteinases-1 promoter in culture-activated rat hepatic stellate cells: regulation by activator protein-1 DNA binding proteins. Hepatology 1999; 29: 839-848.

12. Martin DC, Sanchez-Sweatman OH, Ho AT, Inderdeo DS, Tsao MS, Khokha R. Transgenic TIMP-1 inhibits simian virus $40 \mathrm{~T}$ antigeninduced hepatocarcinogenesis by impairment of hepatocellular proliferation and tumor angiogenesis. Lab Invest 1999; 79: 225-234.

13. Akira $S$, Taga $T$, Kishimoto T. Interleukin- 6 in biology and medicine. Adv Immunol 1993; 54: 1-78.
14. Smart DE, Vincent KJ, Arthur MJ, Eickelberg O, Castellazzi M, Mann J, et al. JunD regulates transcription of the tissue inhibitor of metalloproteinases- 1 and interleukin- 6 genes in activated hepatic stellate cells. J Biol Chem 2001; 276: 24414-24421.

15. Ward NS, Waxman AB, Homer RJ, Mantell LL, Einarsson O, Du Y, et al. Interleukin-6-induced protection in hyperoxic acute lung injury. Am J Respir Cell Mol Biol 2000; 22: 535-542.

16. Kaptein A, Paillard V, Saunders M. Dominant negative stat3 mutant inhibits interleukin-6-induced Jak-STAT signal transduction. J Biol Chem 1996; 271: 5961-5964.

17. He TC, Zhou S, da Costa LT, Yu J, Kinzler KW, Vogelstein B. A simplified system for generating recombinant adenoviruses. Proc Natl Acad Sci U S A 1998; 95: 2509-2514.

18. Sato $Y$, Rifkin DB. Inhibition of endothelial cell movement by pericytes and smooth muscle cells: activation of a latent transforming growth factor-beta 1-like molecule by plasmin during co-culture. J Cell Biol 1989; 109: 309-315.

19. Bugno M, Graeve L, Gatsios P, Koj A, Heinrich PC, Travis J, et al. Identification of the interleukin-6/oncostatin $M$ response element in the rat tissue inhibitor of metalloproteinases-1 (TIMP-1) promoter. Nucleic Acids Res 1995; 23: 5041-5047.

20. Khatiwala CB, Peyton SR, Putnam AJ. Intrinsic mechanical properties of the extracellular matrix affect the behavior of pre-osteoblastic MC3T3-E1 cells. Am J Physiol Cell Physiol 2006; 290: C1640C1650.

21. Ingber DE. Mechanical signaling and the cellular response to extracellular matrix in angiogenesis and cardiovascular physiology. Circ Res 2002; 91: 877-887.

22. lizasa T, Fujisawa T, Suzuki M, Motohashi S, Yasufuku K, Yasukawa $\mathrm{T}$, et al. Elevated levels of circulating plasma matrix metalloproteinase 9 in non-small cell lung cancer patients. Clin Cancer Res 1999; 5: $149-153$.

23. Ming SH, Sun TY, Xiao W, Xu XM. Matrix metalloproteinases-2, -9 and tissue inhibitor of metalloproteinase-1 in lung cancer invasion and metastasis. Chin Med J 2005; 118: 69-72.

24. Gomez DE, Alonso DF, Yoshiji H, Thorgeirsson UP. Tissue inhibitors of metalloproteinases: structure, regulation and biological functions. Eur J Cell Biol 1997; 74: 111-122.

25. Chirco R, Liu XW, Jung KK, Kim HR. Novel functions of TIMPs in cell signaling. Cancer Metastasis Rev 2006; 25: 99-113.

26. Luparello C, Avanzato G, Carella C, Pucci-Minafra I. Tissue inhibitor of metalloprotease (TIMP)-1 and proliferative behaviour of clonal breast cancer cells. Breast Cancer Res Treat 1999; 54: 235-244.

27. Oelmann E, Herbst H, Zuhlsdorf M, Albrecht O, Nolte A, Schmitmann $C$, et al. Tissue inhibitor of metalloproteinases 1 is an autocrine and paracrine survival factor, with additional immune-regulatory functions, expressed by Hodgkin/Reed-Sternberg cells. Blood 2002; 99: 258-267.

28. Murphy FR, Issa R, Zhou X, Ratnarajah S, Nagase H, Arthur MJ, et al. Inhibition of apoptosis of activated hepatic stellate cells by tissue 
inhibitor of metalloproteinase-1 is mediated via effects on matrix metalloproteinase inhibition: implications for reversibility of liver fibrosis. J Biol Chem 2002; 277: 11069-11076.

29. Vorotnikova E, Tries M, Braunhut S. Retinoids and TIMP1 prevent radiation-induced apoptosis of capillary endothelial cells. Radiat Res 2004; 161: 174-184.

30. Liu XW, Taube ME, Jung KK, Dong Z, Lee YJ, Roshy S, et al. Tissue inhibitor of metalloproteinase-1 protects human breast epithelial cells from extrinsic cell death: a potential oncogenic activity of tissue inhibitor of metalloproteinase-1. Cancer Res 2005; 65: 898-906.

31. Lambert E, Boudot C, Kadri Z, Soula-Rothhut M, Sowa ML, Mayeux $\mathrm{P}$, et al. Tissue inhibitor of metalloproteinases-1 signalling pathway leading to erythroid cell survival. Biochem J 2003; 372: 767-774.

32. Bertaux B, Hornebeck W, Eisen AZ, Dubertret L. Growth stimulation of human keratinocytes by tissue inhibitor of metalloproteinases. $J$ Invest Dermatol 1991; 97: 679-685.

33. Fata JE, Leco KJ, Moorehead RA, Martin DC, Khokha R. Timp-1 is important for epithelial proliferation and branching morphogenesis during mouse mammary development. Dev Biol 1999; 211: 238254.

34. Streetz KL, Wustefeld T, Klein C, Manns MP, Trautwein C. Mediators of inflammation and acute phase response in the liver. Cell Mol
Biol (Noisy-le-grand) 2001; 47: 661-673.

35. Michalopoulos GK, DeFrances MC. Liver regeneration. Science 1997; 276: 60-66.

36. Takizawa H, Ohtoshi T, Ohta K, Yamashita N, Hirohata S, Hirai K, et al. Growth inhibition of human lung cancer cell lines by interleukin 6 in vitro: a possible role in tumor growth via an autocrine mechanism. Cancer Res 1993; 53: 4175-4181.

37. Danforth DN Jr, Sgagias MK. Interleukin-1 alpha and interleukin-6 act additively to inhibit growth of MCF-7 breast cancer cells in vitro. Cancer Res 1993; 53: 1538-1545.

38. Badache A, Hynes NE. Interleukin 6 inhibits proliferation and, in cooperation with an epidermal growth factor receptor autocrine loop, increases migration of T47D breast cancer cells. Cancer Res 2001; 61: 383-391.

39. Zeng XH, Fan XJ. Apoptosis of liver cancer cells BEL-7402 induced by IL- 6 and its calcium signal transduction pathway. Acta Laser Bio Sinica 2001; 10: 55-61.

40. Silacci P, Dayer JM, Desgeorges A, Peter R, Manueddu C, Guerne PA. Interleukin (IL)-6 and its soluble receptor induce TIMP-1 expression in synoviocytes and chondrocytes, and block IL-1-induced collagenolytic activity. J Biol Chem 1998; 273: 13625-13629. 